Itinéraires Itinéraires

Littérature, textes, cultures

2009-2 | 2009

Caraïbe et océan Indien

\title{
Les fantômes de Maurice et la littérature
}

\section{Guillaume Bridet}

\section{OpenEdition}

\section{Journals}

Édition électronique

URL : http://journals.openedition.org/itineraires/304

DOI : 10.4000/itineraires.304

ISSN : 2427-920X

Éditeur

Pléiade

Édition imprimée

Date de publication : 1 juillet 2009

Pagination : 127-146

ISBN : 978-2-296-09639-4

ISSN : 2100-1340

Référence électronique

Guillaume Bridet, "Les fantômes de Maurice et la littérature », Itinéraires [En ligne], 2009-2 | 2009, mis en ligne le 01 juillet 2009, consulté le 02 mai 2019. URL : http://journals.openedition.org/ itineraires/304 ; DOI : 10.4000/itineraires.304

Ce document a été généré automatiquement le 2 mai 2019.

\section{(c) (i) () $\Theta$}

Itinéraires est mis à disposition selon les termes de la licence Creative Commons Attribution - Pas d'Utilisation Commerciale - Pas de Modification 4.0 International. 


\title{
Les fantômes de Maurice et la littérature
}

\author{
Guillaume Bridet
}

1 Prétendre que Barlen Pyamootoo n'est pas un auteur mauricien serait sans doute excessif. Avant la publication de Salogi's, texte autobiographique paru en 2008 et qui donne un certain nombre d'informations sur lui, l'écrivain ne ménageait pourtant pas sa peine. Il n'est que de considérer la couverture des deux livres de fiction qu'il avait donnés jusqu'alors, Bénarès en 1999 et Le Tour de Babylone en 2002. Il y a d'abord les nom et prénom de l'auteur lui-même, qui nous orientent vaguement vers l'espace indien; le nom de l'éditeur, L'Olivier, qui le fixe ensuite à Paris; les titres enfin, qui finissent de nous égarer: Bénarès évoque apparemment une ville du Nord de l'Inde, quand Le Tour de Babylone attire au contraire notre attention, moins vers l'Irak actuel, que vers la très ancienne Mésopotamie. Et il ne faut pas compter sur la vignette photographique qui orne chacune des deux couvertures pour en apprendre davantage, tant elle se caractérise par son absence de signes référentiels précis : dans la pénombre d'une porte où un chat assoupi a trouvé refuge, celle de Bénarès figure un homme de dos à côté d'une bicyclette et appuyé à une bicoque en bois ; sur celle du Tour de Babylone, on aperçoit un mur sali percé d'une porte et d'une fenêtre et devant lequel se détachent un homme, un enfant et une balançoire métallique qu'occupent deux autres petites filles. Un auteur de l'Antiquité, Barleen Pyamootoo, un migrant passé par voie de terre de l'Asie du Sud à la France ou un métis dont le sang coulerait de la Seine à l'Euphrate et au Gange ? Il faut retourner le livre et lire la quatrième de couverture pour que soit mis un terme à notre égarement et que l'origine comme la résidence mauriciennes de l'auteur soient mentionnées - du coup un peu déceptives dans leur clôture identitaire, mais attirant aussi notre attention sur une île dont la population, fruit de vagues d'émigration successives, a pour principale caractéristique d'être extraordinairement mêlée. On aurait tort cependant de s'arrimer intérieurement à cette mince certitude et de considérer l'appartenance nationale de l'auteur comme le prisme exclusif ou seulement central de la lecture de ses deux récits. C'est en effet l'ensemble de leurs caractéristiques qui semble déjouer comme 
volontairement toute tentative de pétrification identitaire et laisser place à des présences dédoublées et fantomatiques - pour que vive la littérature.

2 Dans Bénarès comme dans Le Tour de Babylone, les conventions du récit réaliste sont clairement transgressées : aucun regard ni aucune parole de surplomb, mais la perception et le discours d'un personnage immergé dans la réalité. Ce n'est pas que les informations soient systématiquement gommées, mais elles passent par la voix d'un narrateur homodiégétique qui ne prend pas la peine de donner toutes les précisions que le lecteur serait en droit d'attendre. L'univers fictionnel reste donc lacunaire.

3 C'est d'abord l'ancrage spatio-temporel qui n'est pas établi avec une grande précision. Dans Bénarès, l'histoire commence « un jour » (Pyamootoo, 1999: 7) sans date, alors que le soleil se lève et que le narrateur, qui a fini de se raser, est en train de s'habiller. Ce début de journée est aussi le début du récit. La description liminaire de sa maison et du village qui l'entoure est fort simple : un chemin de terre, la mer, une plage, un dispensaire, un bureau de poste, une école - rien qui permette de localiser précisément le lieu. Les personnages passent en taxi dans des localités portant les noms peu caractéristiques de Port-Louis, Pointe aux Sables, Sainte Croix ou Curepipe ; eux-mêmes vivent à Bénarès, mais dans un pays qu'on n'est pas certain de pouvoir identifier comme l'Inde, dans la mesure où les toponymes précédents ne concordent guère. Les « roupies » (Ibid. : 10) dont il est question assez vite orientent bien l'histoire en Asie du Sud et dans l'océan Indien, mais elles ne permettent pas de la localiser précisément à Maurice et ne mettent ainsi pas de terme définitif à l'incertitude. Le Tour de Babylone peut d'abord sembler plus précis dans son ancrage spatio-temporel. Le narrateur relate une longue déambulation dans divers lieux d'un pays rapidement identifié comme étant l'Irak grâce à la présence de noms de villes bien connues, comme Bagdad, Mossoul ou Kerbela. Quand les personnages évoquent une guerre récente et quand le narrateur constate par lui-même les destructions, on comprend, si l'on considère aussi la date de parution du livre, que l'histoire prend place après la première guerre du Golfe, dans cet après-guerre qu'on sait aujourd'hui être un entre-deux-guerres. Le trouble ici n'est pas tant dans l'imprécision référentielle que dans l'absence de conformité, au moins apparente, entre le titre et l'histoire relatée, puisque, si la fin du chapitre II relate bien l'arrivée à Babylone, le début du chapitre III se déroule à Bagdad. Le tour de Babylone a peut-être lieu mais il n'est pas raconté par le narrateur.

4 Les conventions du récit réaliste sont de la même manière doucement subverties concernant les personnages. Ces derniers n'ont pas de nom, la plupart du temps pas d'âge, pas davantage de situation familiale bien définie. Du narrateur de Bénarès, on sait seulement qu'il rentre «du travail » et il faut attendre la seconde moitié du récit pour apprendre qu'il est "aide-mécanicien dans un garage »; son ami Mayi est rapidement identifié comme "pêcheur " (Ibid. : respectivement 10, 55 et 8), mais la quasi-anagramme que constitue son prénom semble surtout renvoyer à sa fonction romanesque d'ami du narrateur ; Jimi est chauffeur de taxi ; les deux femmes que ces trois hommes fréquentent l'espace d'une soirée, Mina et Zelda, sont des prostituées dont la biographie reste extrêmement lacunaire. Dans Le Tour de Babylone, l'identité des personnages est encore plus imprécise : à aucun moment n'est indiqué d'où vient le narrateur ni ce qu'il est venu faire en Irak : journaliste, voyageur, touriste ? La seule chose certaine, c'est qu'il a des « enfants » (Pyamootoo, 2002: 44), mais on ne peut rien dire ni de l'endroit où ils vivent ni de leur mère. On ne saura pas davantage qui est cet Hassan qui accompagne le narrateur dans sa visite de Bagdad. Et cette Salma, pourquoi marche-t-elle avec lui dans 
les rues de la ville? «Elle me prend la main, joue avec nos doigts, puis soudain, se blottit contre moi comme une enfant, en s'accrochant à mes épaules » (Ibid. : 93). L'intimité entre les deux personnages semble évidente mais il est difficile de savoir jusqu'où elle va et ce qu'ils ont exactement vécu ensemble.

5 À ce cadre spatio-temporel troué et à ces personnages flous correspond de manière concordante une histoire réduite au minimum. Dans Bénarès, deux amis conduits par un chauffeur de taxi de leur connaissance recherchent et trouvent deux prostituées pour passer la nuit avec elles. Le récit est divisé en six chapitres: dans les deux premiers aboutit la quête des femmes; dans les quatre suivants a lieu le retour en voiture vers Bénarès. Le récit s'apparente à une espèce de road movie mais décalé dans l'insignifiant, sans action autre que minuscule. N'ont lieu que des gestes simples et des conversations sommaires. Un personnage demande à l'autre une cigarette; pose une question; le regarde avant de détourner les yeux. L'histoire s'interrompt quand tout devrait commencer. À la pittoresque et attendue partie de jambes en l'air tropicale se substitue en effet une série de conversations entrecoupées de silence dans l'habitacle confiné de la voiture. Le Tour de Babylone a beau se dérouler dans un pays tout juste sorti de la guerre, il déjoue aussi l'attente, puisqu'il constitue moins un reportage qu'il ne relève du genre plus disparate de la chose vue (et entendue). Le narrateur marche dans Bagdad (chapitre I), puis il prend la route qui conduit à Samarra (chapitre II), avant de déambuler de nouveau dans la capitale irakienne jusqu'à son départ imminent en train pour Amman (chapitres III et IV). Il voit des choses, assiste à des scènes, rencontre des Irakiens qui lui parlent de leur pays marqué par le désordre, la saleté et la misère : monceaux de gravats, bouteilles en plastique, chiens errants, hommes qui fument avec ennui ou qui s'insultent, militaires et policiers omniprésents, enfants livrés à eux-mêmes dans les rues. Ce que le narrateur décrit à la volée, comme par une suite de croquis ou d'enregistrements pris sur le vif, ce sont les ravages d'un après-guerre et la vie comme elle continue d'aller dans une quotidienneté de privations (le pays est sous embargo) et de tyrannie (Saddam Hussein est encore au pouvoir). Bénarès se situe ainsi dans l'avant et Le Tour de Babylone dans l'après de ce qu'on pourrait considérer comme l'événement attendu, ici de l'ordre de l'histoire collective, là plus intime.

6 Ni sexe à Bénarès, ni guerre à Bagdad, mais quoi alors? Selon un usage littéraire bien répertorié en France depuis les années 1950 - et que l'enseignant puis l'éditeur qu'est aussi Barlen Pyamootoo n'ignore sans aucun doute pas -, l'écriture d'une aventure laisse la place à l'aventure d'une écriture et s'inscrit dans le cas présent dans une tradition minimaliste bien française dont les Éditions de Minuit sont aujourd'hui encore le lieu de publication privilégié. On pense ainsi - et des allusions de Bénarès le confirment ${ }^{1}$ - à Duras et surtout à Beckett ou encore au Lendemain de fête (1993) de Jacques Serena.

7 Ce que cherche à maintenir le narrateur modeste de chacun des deux récits de Barlen Pyamootoo, c'est une perception immédiate qui ne prenne jamais de hauteur ni physique, ni conceptuelle, ni surtout idéologique.

Je laisse la voiture dans la plaine à l'ombre des fleurs sauvages et je grimpe la colline en courant, je veux d'abord voir Babylone de haut. Mais je dois m'arrêter à mi-côte : le sentier s'est rétréci jusqu'à disparaître devant un rocher couvert de mousse. [...] je m'accroupis par terre et contemple la cité qui semble sortie tout droit des temps bibliques: elle est bâtie comme une forteresse, avec ses hautes flèches effilées qui rognent sur le ciel. (Ibid. : 52) comme s'il était impossible, ou simplement comme s'il n'était pas souhaitable de 
contempler Babylone de haut, d'envisager la réalité avec une position de surplomb. Le narrateur occupe un point de vue comparable à celui de Fabrice à Waterloo, mais d'un Fabrice qui aurait pris le parti de son ignorance, accepterait d'avoir perdu ses repères et se contenterait d'une attention de myope à ce qui se situe au plus proche. Cette attention de portée réduite mais précise est salutaire, car, à tout prendre, la réalité telle qu'elle est construite dans Bénarès et dans Le Tour de Babylone n'est pas seulement lacunaire ou déceptive, elle offre des apparences essentiellement trompeuses. Ce n'est pas d'évanescence spatio-temporelle, personnelle ou événementielle qu'il faut parler, mais bien d'un jeu de fausses pistes qui constituent autant de mises en garde contre la tendance à percevoir la réalité à travers les lunettes lointaines et déformantes du stéréotype: la sexualité sous les tropiques est nécessairement débridée, les classes populaires sont des classes vulgaires et dangereuses, les femmes sont de petites et faibles choses, les musulmans sont des intégristes, etc.

Les deux récits piégés offrent bien des exemples venant invalider ce type d'idées reçues. Au bistrot de la gare de Bagdad où il vient d'entrer avec Salma, le narrateur du Tour de Babylone s'apprête à boire de l'alcool en terre musulmane en compagnie d'une femme arabe : "On s'assied au comptoir près d'une bande d'adolescents et on commande une bière, un cocktail maison et des beignets aux légumes » (Ibid. : 97). Au Mayi de Bénarès qui lui explique qu'il aime jouer au football, la prostituée Mina répond contre toute attente qu'elle est aussi une footballeuse: «J'ai toujours été dans les buts» (Pyamootoo, 1999: 50). La négation des stéréotypes s'étend en fait à toutes les associations d'idées trop rapides qui masquent la réalité. Quand les personnages masculins s'arrêtent à SainteCroix face à «une maison [...] illuminée » devant laquelle s'étend « un jardin avec des roses de plusieurs couleurs » (Ibid.: 19), le narrateur se livre à une sémiologie appliquée de l'habitat qui révèle à quel point il ne faut pas se fier aux apparences. Cherchant à se figurer ses habitants et quels métiers ils exercent, il pense à des personnes travaillant « dans une banque ou dans un ministère », ou encore « dans les affaires » (Ibid. : 19-20). Il se trompe. "C'est ici qu'habite Ma Tante", annonce en effet Jimi devant ce jardin où chaque rose déploie "son halo de clarté comme une pierre précieuse » (Ibid.: 20). Toute rose, la vie de souteneuse - une vie pavillonnaire comme une autre? Pas pour autant - et un stéréotype à peine écarté, c'est un lieu commun qui vacille, qui voudrait qu'à un extérieur magnifique corresponde un intérieur de même nature. Car la « pièce [...] située à l'arrière de la maison » et dans laquelle se rendent les personnages déjoue l'attente suscitée par le jardin : elle est teinte de «moisi », sent « le ciment » et n'est décorée que de "posters à moitié décollés » (Ibid. : 23). Cette rapide description laisse percer un léger pathétique, quand la toute fin du chapitre en reprend les principaux éléments à travers le regard triste de la maîtresse des lieux : « elle a regardé d'un air désœuvré les posters, le sol, les verres et les fauteuils vides" (Ibid. : 25). Une maquerelle n'a décidément pas exactement la vie satisfaite d'une petite-bourgeoise pavillonnaire (ou ce sont les bourgeoises qui sont tristes comme des maquerelles).

Mais ce qui frappe-surtout dans Bénarès-, c'est l'extrême délicatesse des relations humaines et des sentiments exprimés ou simplement éprouvés par les personnages. Lorsque le narrateur se lave puis passe « un jean et un T-shirt avec "Miami" écrit dessus " avant de préciser : « J'aime les vêtements où il y a quelque chose à lire, c'est ce que je lis le plus» (Ibid.: 11), le lecteur comprend qu'il n'a pas affaire aux hautes couches de la société. Mais l'association automatique de la modestie culturelle et économique et de la vulgarité, voire de la violence, est aussitôt battue en brèche. Mayi, dont le football semble 
la grande passion, ne se refuse-t-il pas à jouer à l'avant - où l'on brille en marquant des buts - et ne demeure-t-il pas plus discrètement à l'arrière en simple signe d'amitié ? « Il y a le gardien, je ne pourrais pas le laisser seul, c'est un ami» (Ibid.: 52). Mayi est un personnage essentiellement généreux qui entend faire profiter le narrateur de l'argent qu'il a gagné aux cartes. Ce dernier le lui rend bien, qui est d'une grande attention à son égard, comme l'indique, dès les premières pages et tout le long du récit, la multiplication des notations le concernant. Le narrateur guette les émotions éprouvées par son ami et s'enquiert de son bien-être: il scrute son visage livide, ses yeux dans lesquels perce la peine, ses paupières alourdies par le sommeil. Les deux hommes se montrent aussi d'une grande délicatesse avec les deux femmes qui ont accepté de monter en voiture avec eux. Pour Mayi, il ne saurait être question de marchander le prix de la nuit, et son sourire n'est pas entamé par l'argent à débourser. Le narrateur n'est pas en reste qui, s'il est mécanicien, n'en a pas moins les mains propres et l'âme, mieux encore, les mots d'un poète, quand il pense à Zelda : «Elle sentait la pomme sure et l'humus des bois, c'était un mélange de sueur et de parfum qui avait des relents de fleurs fanées, tombées au fond d'un vase, oubliées » (Ibid. : 54).

11 Si toute trivialité n'est pas absente, il n'en reste pas moins que, dans ce monde de douceur suspendue entre les êtres que configure le récit de Bénarès, les stéréotypes classistes, racistes et misogynes sont mis à dure épreuve. Ce qui aurait pu être l'histoire d'une virée nocturne sur fond de désir sexuel apparait surtout comme une initiation de la sensibilité : un partage du sensible qui ne peut aller sans un décrassage de tous les mots qu'un prétendu savoir social - composé essentiellement d'opinions désindividualisantes - pose sur les objets et les êtres. La vie des personnages de Barlen Pyamootoo est faite de riens partagés, plaisirs minuscules intensément vécus et qui signent l'être ensemble - petites gorgées de rhum et bonbons à la menthe ou questions sans queue ni tête sur le sexe des étoiles. "Jamais je ne me suis senti aussi bien » (Ibid. : 29), confie Mayi au narrateur. C'est toute la modestie d'une certaine façon d'être au monde et aux autres qui apparait ici, taiseuse et cependant bienheureuse dans sa fraîcheur et son épiphanie - toutefois sans innocence, on va le voir.

Cette épiphanie du sensible, c'est sans aucun doute dans Le Tour de Babylone qu'elle se donne à lire et à sentir avec la plus grande acuité. Comme l'explique le narrateur en une phrase décisive commentant son propre récit, « il y a la beauté même sur des routes qui ne mènent nulle part » (Pyamootoo, 2002: 16). Compte en effet moins la réalité de la misère irakienne que la réalité dans sa profusion et sa banalité, suite d'objets et d'individus qui se succèdent sans démonstration ni même sans hiérarchie apparentes, mais qui tous suscitent une forme d'émerveillement - sans que cet émerveillement soit le moins du monde fondé sur les lieux communs de l'orientalisme, ni sur leur retournement d'ailleurs : ni Orient doré de pacotille, ni Orient misérable et glorifié pour cela même, juste une réalité éprouvée au plus près et présente pour elle-même. Se promenant dans les rues de Samarra et dans les chemins qui montent vers les palmeraies, le narrateur éprouve la sensation d'un monde renouvelé par l'attention extrême qu'il lui porte :

Par moments mes yeux se ferment de béatitude, je pense à mon voyage, prêt pour l'aventure, et à d'autres je me laisse bouleverser par des images qui peuvent paraitre fugaces ou anodines : des pierres gravées au-dessus des portes, un homme qui se bat tout seul dans l'herbe, coiffé d'un turban au tissu rayé, des ouvriers écroulés le long d'un mur et au coin d'un sentier une barque que répare un calfat en tapant du pied. (Ibid. : 48) 
13 On pense autant au Rimbaud d'Une saison en enfer évoquant son goût pour les « peintures idiotes, dessus de portes, décors, toiles de saltimbanques, enseignes, enluminures populaires » (Rimbaud, 1972 : 106) qu'au Nicolas Bouvier de L'Usage du monde, voyageant des Balkans à la frontière indienne avec le souci de donner sa juste place à chaque chose et à chaque personne rencontrée puis de délivrer par l'écriture leur poids de présence. La poésie n'a pas de niche; elle court sur les routes du monde. Le retournement ou la neutralisation des stéréotypes laisse apparaître une nouvelle réalité - qui n'est pas celle du réalisme, qui en est même l'exact opposé, réalité construite, non sur la mobilisation des lieux communs qui, dans leur facilité et leur paresse, simplifient et élaguent la réalité au point de parfois la trahir, mais sur une attention sans faille à notre propre sensibilité. La fonction très profonde de cette mise à mal des procédés réalistes suffirait pour affirmer que la littérature de Barlen Pyamootoo n'est pas désengagée et repliée dans une affirmation minimaliste purement esthétique mais qu'elle comporte à sa manière une dimension éminemment politique.

L'auteur ne se place pas dans un volontarisme identitaire, tel qu'on peut le lire dans le sillage de la coolitude - démarquée de la négritude - du poète mauricien Khal Torabully². Il n'est pas même comparable à certains auteurs d'origine indienne qui, comme le Réunionnais Firmin Lacpatia ou le Guadeloupéen Ernest Moutoussamy ${ }^{3}$, se font l'émanation d'une voix collective, ancestrale et attachée à décrire précisément les croyances, les rites, la langue ou encore l'histoire d'une communauté qui trouverait dans ce point d'appui la garantie d'une continuité identitaire lui permettant de s'inscrire dans un présent métissé : on ne trouve aucune de ces caractéristiques qui définissent un « ethnotexte » (Magdelaine-Andrianjafitrimo, 2004b : 94) dans Bénarès et dans Le Tour de Babylone. L'auteur n'écrit pas, comme l'écrivain de langue hindi Abhimanyu Unnuth ${ }^{4}$ ou comme l'écrivain de langue française Nathacha Appanah-Mouriquand ${ }^{5}$, un roman de l'engagisme et des immigrants qui, fuyant la famine et loin d'imaginer l'exploitation qui les attendait, quittèrent l'Inde à partir du milieu des années 1830 pour s'embaucher dans les plantations de Maurice ${ }^{6}$. Le passage du taxi de Bénarès à Pointe aux Sables aurait pu être l'occasion de décrire plus précisément ce quartier ghetto et, en particulier, la présence d'une partie des exilés de la communauté des Chagossiens qui y a trouvé refuge dans des conditions extrêmement précaires depuis le début des années 19707. Mais Barlen Pyamootoo n'est pas Shenaz Patel, et Bénarès n'est pas Le Silence des Chagos. Il en va de même du passage par Port-Louis. Barlen Pyamootoo aurait pu, comme Ananda Devi dans Rue La Poudrière, décrire plus précisément cette ville de misère où les Créoles souffrent d'une ségrégation à la fois économique et sociale et où la prostitution tient une place importante. Mais rien de cela : seulement quelques personnages sur le fond d'un décor urbain et social mal défini.

Pas de pittoresque, pas de discours, pas même de mise en relation explicite de personnages d'origines culturelles différentes, mais la mise en cause radicale et d'une bien plus grande portée de toute assignation identitaire et la mise en déroute des codes de perception les plus communs: telle est la façon très particulière qu'a Barlen Pyamootoo de penser la possibilité d'une société multiculturelle qui se trouve dans la nécessité d'accepter la variété de son peuplement. On ne trouve rien dans ses deux courts romans de l'humanisme interculturel du Namasté de Marcel Cabon réhabilitant l'image particulièrement malmenée ${ }^{8}$ de l'Indo-Mauricien ou même de la réflexion d'une MarieThérèse Humbert dans À l'autre bout de moi explorant les limites et les possibilités des négociations transculturelles entre Mauriciens d'origines indienne et européenne. De ce 
point de vue, l'auteur se place clairement du côté d'une créolisation entendue, non pas seulement comme une catégorie historique correspondant à la réalité géographique et historique de Maurice, mais, plus largement et d'un point de vue théorique proche par exemple de celui de Homi Bhabha, comme une contestation de tous les discours identitaires glorifiant la racine et les liens du sang et comme constat - non pas même louange - d'une identité essentiellement hybride et complexe des individus comme des cultures et des sociétés.

Il faut ajouter que le retournement et la neutralisation des stéréotypes n'impliquent pas une fuite devant la réalité la plus brutale qui soit. Qui dit épiphanie de la banalité ne dit pas disparition de la violence de l'histoire et des rapports sociaux. Barleen Pyamootoo n'est pas Delerme - ni père, ni fils -, et la gorgée de rhum ne remplace pas la gorgée de bière pour occulter les traces d'une souffrance, essentiellement économique dans Bénarès, à la fois économique et politique dans Le Tour de Babylone. Ici, des soldats suicidaires, des villageois déplacés et entassés dans des camps de fortune; là, le chômage, la prostitution et le travail des enfants ; partout : des hommes considérés comme "du poisson pourri » (Pyamootoo, 2002: 100). Dénonciation de la misère, de la guerre, de la tyrannie? Sans aucun doute. Mais c'est dans Le Tour de Babylone qu'apparait de la manière la plus explicite le point d'impact central que les deux récits de Barlen Pyamootoo entendent toucher: moins la souffrance économique et politique des hommes que les représentations mensongères qu'en donnent certains pouvoirs. La mise en cause du réalisme et l'épiphanie d'une nouvelle réalité doivent conduire le lecteur à prêter attention au monde qui l'entoure ; elles constituent aussi une critique des discours de propagande politique.

Le narrateur qui marche dans Bagdad ne cesse en effet de souligner à quel point le tyran est présent dans la ville par le biais des portraits qui le représentent.

On s'arrête au coin de la rue et on regarde un portrait de Saddam accroché à un réverbère. «Il est partout », me dit Hassan, « en train de refaire l'Histoire. » Il porte une chemise à carreaux et un pantalon retenu à la taille par une ficelle. Un râteau à la main, il sourit au milieu des fleurs et des pommes grosses comme des melons. Je pense à quelqu'un au village qu'on appelle Saddam à cause de sa moustache et de sa grande gueule. (Ibid. : 21)

La touche burlesque finale, qui ravale le dictateur sanguinaire au statut de coq de village inoffensif, ne doit pas dissimuler l'essentiel : cet homme omniprésent impose à tous les citadins une image de lui-même en jardinier patelin censé transformer l'Irak en un paradis terrestre - et l'on sait toute la dimension sacrée du jardin dans la culture arabomusulmane -, mais qui inverse la réalité d'une dictature cruelle qui a davantage à voir avec l'enfer. Barlen Pyamootoo rejoint ici, et peut-être sciemment, les réflexions que Milan Kundera consacre au kitsch-toujours potentiellement totalitaire-comme «besoin de se regarder dans le miroir du mensonge embellissant et de s'y reconnaître avec une satisfaction émue » (Kundera, 1986 : 165). Il y a, d'un côté, le discours imagé de «l'univers totalitaire » qui recouvre la réalité d'images falsificatrices, de l'autre, l'absence tendancielle de discours d'un roman s'efforçant de laisser advenir la réalité dans sa diversité et témoignant, par là même, d'une «sagesse de l'incertitude " (Ibid.: respectivement 29 et 21). Cela ne signifie certes pas que le réalisme littéraire soit réductible à la propagande totalitaire, mais que l'un comme l'autre ont en commun une prétention monosémique et la négation d'une sensibilité nécessairement relativiste et respectueuse de la complexité, voire de l'opacité du monde. 
20 On aurait cependant tort de s'arrêter à cette lecture simplement humaniste des deux récits de Barlen Pyamootoo. La lutte contre la réalité des conventions réalistes ou de l'idéologie politique au profit d'une autre réalité construite par un renouvellement de la sensibilité n'est pas sans faille. Elle ne va pas sans susciter des troubles de la perception si inquiétants qu'ils conduisent nos récits au bord du genre fantastique.

21 Dans Le Tour de Babylone, les traces de ce trouble sont relativement modestes mais ils touchent le point décisif de l'identification des objets dans leur différence. Les instants ne sont pas rares en effet où le narrateur ne peut faire autrement, évoquant la réalité irakienne, que de la renvoyer à une autre réalité pour le moins surprenante. «Cet homme qui marche le dos voûté » ressemble à «un Bavarois en costume: short, bretelles et chapeau »; les photographies de Babylone reconstruite font penser à "Cinecittà »; un dialogue entre deux hommes qui ne parlent pas de la même chose est comme « un poème de Pessoa » (Pyamootoo, 2002 : respectivement 24,64 et 82), etc. Il ne faut pas négliger la dimension humoristique de telles comparaisons qui, arrachant l'histoire à son contexte immédiat, font signe vers la culture que partagent les lecteurs occidentaux susceptibles de lire les romans de Barlen Pyamootoo. Mais elles ne sont pas seulement le signe d'une connivence recherchée. Ces rapprochements orchestrent un décloisonnement géographique qui universalise la réalité dans sa banalité. Le narrateur le reconnaît luimême : «Et ils ne sont pas si différents de ceux de mon pays, les gamins qui jouent à la guerre, les fenêtres munies de grillages en fer et les regards braqués sur les filles, ça me donne même l'illusion de retourner sur le lieu de mon enfance » (Ibid. : 30).

Les effets politiques de ce type de comparaisons sont difficiles à nier, et la leçon en paraît limpide : tout est pareil sous le soleil et, qu'on soit d'ici ou d'ailleurs, on est confronté à la même réalité - propos humaniste une fois encore, de portée finalement assez faible dans son unanimisme et qu'il convient de dépasser pour considérer le pouvoir d'inquiétude que, dans une proximité avec l'œuvre de Borges, recèlent ces rapprochements intempestifs. Si tout lieu, tout objet ou tout personnage est susceptible d'en évoquer un autre, cela signifie, non seulement qu'il est peu singularisé et n'a pas de réalité qui lui soit propre, mais peut-être aussi finalement qu'il n'a pas de réalité du tout. De la déterritorialisation à l'absence de territoire ou de la désindividualisation à l'absence d'individualité, il n'y a pas loin. Pour atténuer l'inquiétude, le narrateur a recours à des motivations de type psychologique ou physiologique courantes dans le genre fantastique : ici, excès « d'images et de souvenirs » qui font perdre le sens des réalités ; là, « des sortes de mirages qui montent à la tête » (Ibid. : respectivement 29 et 91). L'inquiétude demeure néanmoins et les apparitions se multiplient : "une vieille fée descend d'un autobus », « un garçon » semble si « beau » que « c'est presque de la magie » et « une femme [...] avec des doigts crochus " (Ibid. : respectivement 21, 32 et 34 ) évoque une sorcière.

23 Si l'on veut saisir le sens de cette dimension fantastique des deux récits dans lesquels l'espace finit par s'assimiler à l'image du «labyrinthe» (Pyamootoo, 1999: 74; 2002 : 77) - image répertoriée d'une perte qui, redoublée d'un récit à l'autre, finit par égarer le lecteur dans une succession de faux-semblants et d'apparitions fantomatiques - c'est vers la seconde moitié de Bénarès qu'il faut se tourner.

24 Le récit a beau ne pas être d'une grande précision référentielle, il n'en reste pas moins que le fil du temps est d'abord clairement scandé : le narrateur revient «vers cinq heures » du travail ; le taxi qui le transporte avec son ami Mayi arrive à Port-Louis « un peu avant sept heures »; ils sont encore à Pointe aux Sables à "presque huit heures » (Pyamootoo, 1999: respectivement 10, 12 et 15). À partir du moment où les cinq 
personnages commencent leur voyage en voiture s'installe néanmoins un grand flou - une dilatation incroyable du temps qu'accompagne un estompement des limites spatiales. Favorisé comme il se doit par la nuit, un paysage fantastique se dessine peu à peu, « du désert à perte de vue » et d'« un village [...] sinistre » à « une des routes les plus désertes que [le narrateur] connaisse » et au ciel où l'on ne voit " pas une étoile, rien que l'obscurité » (Ibid. : respectivement 55, 61, 62 et 66). Dans le contexte propice de la nuit, l'hyper-esthésie du narrateur révèle une réalité troublée où tout fait signe vers un ailleurs inquiétant. Dans les champs, le narrateur voit "des silhouettes» et il entend "toutes sortes de bruits [...] aussi mystérieux qu'un idiome obscur »: «J'ai pensé à des taupes, à des chauves-souris et à tous ceux qui vivent dans le noir. J'ai même pensé à des vampires et aux cris que ça pouvait pousser» (Ibid.: 62). Entre les conversations entrecoupées par le sommeil des uns et des autres apparaissent le long de la route « des ombres grises qui [rasent] les murs et les façades. [...] Des revenants, je me suis dit, la nuit est peuplée de revenants» (Ibid.: 85). Quand « des cris » retentissent, qui ressemblent à " des couinements, brefs et plutôt perçants », le narrateur évoque cette fois « un animal blessé » et Jimi renchérit avec l'explication, qui surprend dans un pays tropical, de « veneurs qui crient pour signaler aux chasseurs la présence d'une bête » (Ibid. : 89).

Une première interprétation possible de ces phénomènes d'altération de la réalité et de l'identité nous renverrait à l'inquiétante étrangeté freudienne qui, comme on le sait, entretient des liens privilégiés avec le genre fantastique. Mais plutôt que de chercher ce qui, dans les récits de l'auteur pourrait nous permettre d'identifier - sans doute sous le signe du désir incestueux et de l'angoisse de castration ${ }^{9}$ - ce « quelque chose qui est pour la vie psychique familier de tout temps, et qui ne lui est devenu étranger que par le processus de refoulement » (Freud, $1985: 246$ ), nous préférons mettre en avant le chemin très particulier par lequel la littérature permet ici de remplacer l'angoisse par la sublimation. Autant Bénarès éclaire le premier aspect, autant c'est dans Le Tour de Babylone qu'apparaît au mieux le second-les deux volumes fonctionnant de ce point de vue comme un diptyque qui marque aussi une évolution: de la mère au père, de Maurice à l'Irak, des présences fantomatiques dans l'espace réduit de la voiture et de l'île à des dédoublements projetés directement dans un monde et une culture élargis - signe d'une extension, non pas de l'intime à l'histoire, mais d'une histoire intime et collective à l'histoire d'autres intimités et d'autres collectivités qui ont aussi à voir avec la violence, la perte et la question de la réparation.

La première page du Tour de Babylone donne à lire en retrait et légèrement décalé sur la droite un petit texte fort intéressant pour éclairer ce qu'il en est de la sublimation :

Quand j'étais un enfant et que je rentrais tard à la maison, mon père me disait : «Quel tour de Babylone as-tu encore fait?» Je ne lui répondais pas, je m'en allais tête baissée vers ma chambre, j'avais tellement honte lorsqu'on me grondait. Car «faire le tour de Babylone » est une expression péjorative. Elle signifie: errer, vagabonder et ainsi dévier de son chemin. (Pyamootoo, 2002: 7)

De cette expression d'un père non identifié dont on ne peut savoir s'il est celui de l'auteur ou du narrateur - incertitude qui constitue la trace ultime des fantômes et qui remet en cause le statut d'exergue qu'on serait tenté de prêter à ce court texte -, va naître un récit, et d'abord en la prenant apparemment au pied de la lettre : faire effectivement le tour de Babylone. Mais ce n'est pas si facile : «Parfois [...] je me demande si ce voyage à Babylone a un sens, je n'arrête pas d'y penser. J'ai presque envie de rebrousser chemin et de rentrer à la maison, mais que vais-je dire à mes enfants? (Ibid. : 44). Il y avait la honte passée inspirée par le père ; il y a à présent la crainte d'une honte future éprouvée devant les 
enfants. La visite de Babylone est d'abord le moyen de prendre le père au mot et, en même temps, de changer le sens de ses mots : non pas un vagabondage, mais un tour, doté d'un but; et surtout : non pas une marche, mais un livre dont on ne saurait même dire s'il est autobiographique et s'il est le fruit d'un voyage réellement effectué. Faire le tour de Babylone, c'est peut-être entrer dans la ville morte mais c'est surtout ne pas le raconter et raconter autre chose : non pas le tour de la ville, mais le détour par lequel l'écriture se donne un autre objet, en l'occurrence les deux villes de Bagdad et de Samarra qui se trouvent de part et d'autre de la ville antique que la narration choisit de contourner - deux villes : image du dédoublement qu'implique cette autre réalité créée par la littérature elle-même. De la tour de Babylone - Babel - au tour de Babylone, on voit bien que l'accès à la créativité littéraire exige de renoncer à toute forme d'accès à l'un, au réel, à l'essence: non pas prendre la parole du père-parler la langue de Dieu-mais inscrire sa parole singulière dans une reprise qui est aussi un déplacement géographique, un déplacement de sens et un déplacement de pratique. Il s'agit d'assumer soi-même une place de père - non pas la place $d u$ père, mais la place d'un père (parmi d'autres) qui seule permet de resémantiser la parole paternelle. L'auteur écrit devant son père, et devant ses enfants. Il a des comptes à rendre, mais il les rend à sa manière - dans une parole qui laisse place aux fantômes de la réalité mais ne se laisse pas réduire au silence par leur présence.

Dans Bénarès et dans Le Tour de Babylone, la présence des ascendants ne se limite néanmoins pas seulement à celle des parents et il n'est du même coup pas certain que les fantômes ressortissent uniquement à la dramaturgie œdipienne et à la possibilité sublimatoire de l'auteur. C'est l'histoire collective de Maurice qui se trouve ici évoquée, histoire d'une île sans population autochtone et peuplée d'individus issus de bassins culturels et linguistiques hétérogènes et soumis, bien souvent, à une acculturation brutale dès leur implantation dans l'île.

Dans Le Tour de Babylone est tout à fait frappante la manière dont le narrateur se montre d'une extrême porosité à l'égard de la dure réalité irakienne: «Je quitte Bagdad avec soulagement, ç'a été une souffrance, tous ces bruits, j'ai dû me soûler plusieurs nuits pour dormir » (Ibid. : 41). Cette porosité va si loin qu'elle le conduit à faire sienne la réalité de ce qu'il voit : «Et les rues sont sales, ça doit faire comme des mares de pétrole quand il pleut, avec tous nos restes qui flottent» (Ibid.: 65). Cet article possessif qui abat la distance entre les Irakiens et un visiteur venu d'on ne sait où est peut-être le fruit d'une certaine culpabilité : celle du visiteur égotiste qui sait, qu'à un moment ou un autre et malgré la compassion qu'il éprouve pour ses semblables, il quittera le pays meurtri et retrouvera une forme de quiétude auprès des siens. L'origine de cette porosité présente d'un homme venu d'ailleurs est cependant à chercher dans le rapport qu'il entretient, non pas avec l'Irak contemporain, mais avec le passé de Maurice.

Cette porosité entre l'ici et l'ailleurs, le présent et le passé, est le motif central des conversations qui ont lieu dans la voiture de Bénarès que conduit un chauffeur psychopompe. Comme l'explique Jimi, les « touristes » qui viennent passer leurs vacances à Bénarès, "des Allemands, des Français, des Italiens et d'autres encore mais moins nombreux", se comportent avec lui fort "gentiment» (Pyamootoo, 1999: 24). Une précision suit, qui renvoie néanmoins les "blancs» à un passé plus cruel : «il n'avait jamais vu ça " (Ibid.). Il y a le présent plutôt doux du tourisme, mais il y a derrière ce moment historique apaisé une autre histoire : celle de l'exploitation de la canne à sucre. Jimi commence le récit de ce passé en évoquant d'abord la présence ancienne d'«un 
moulin à sucre » sur le site de Bénarès, dont l'activité s'est interrompue il y a trente ans et dont on ne voit plus à présent que « la cheminée » qui « ressemble à un monument aux morts depuis que les propriétaires l'ont retapée » (Ibid. : 42-43). Cette restauration du patrimoine industriel n'est pas hommage rendu aux travailleurs qui autrefois firent vivre le lieu mais glorification des patrons - sans aucun doute blancs, mais la chose n'est pas précisée-, puisqu'ils l'ont entreprise "pour qu'on se souvienne d'eux comme des bâtisseurs ", alors qu'« ils ont fermé le moulin et mis au chômage tout un village [...] pour augmenter leurs profits » (Ibid. : 44). Il y a, d'un côté, un chauffeur de taxi qui s'efforce de faire revivre les morts, de l'autre, des Blancs, qui, en bon Saddam Hussein tropicaux - dictateurs et planteurs, c'est une même engeance -, écrivent et représentent l'histoire à leur manière en ne pensant qu'à leur propre célébration et en effaçant du paysage le souvenir des ouvriers.

31 À ce drame de la restructuration industrielle qui a poussé nombre d'habitants du village de Bénarès à partir ailleurs, s'en ajoute un autre plus ancien qui, s'il a lui aussi à voir avec l'histoire coloniale, ne peut être explicitement nommé par le narrateur qui l'évoque à mots couverts au cours de sa conversation avec Zelda. Car « ça s'est fait il y a longtemps, il y a plus de deux cents ans ", "c'est trop vieux pour qu'on s'en souvienne tout à fait " ( Ibid. : 79). Le manque d'archives, détenues par les maitres blancs, se fait ici sentir, qui empêche le discours de raconter exactement l'histoire de la migration plus ou moins forcée vers Maurice de travailleurs que tout porte-mais rien n'est explicitement signalé - à identifier comme indiens. Nulle trace ne demeure de cet événement enfoui, si ce n'est celle du toponyme. Le narrateur précise en effet « qu'en Inde aussi [...] il y a un endroit qui s'appelle Bénarès », et il ajoute qu'il y a été «l'année dernière [...] au mois d'août » (Ibid. : 64). Ce projet de retournement de préséance entre la célèbre ville indienne et l'obscur village sans pays où vit le narrateur est même au cœur de ce voyage sur les bords du Gange. Ayant expliqué que « dans [son] pays aussi, il existe un endroit qui porte le même nom », le narrateur poursuit de la sorte : «Et parfois, [...] je me demande si je ne suis pas allé à Bénarès rien que pour eux, pour qu'un jour ils fassent le voyage eux aussi » (Ibid. : 74-76). Ce dont il s'agit ici, ce n'est pas seulement faire reconnaitre un lieu ignoré de tous - et d'autant moins que des touristes viennent déjà à Bénarès -, mais le faire précisément reconnaître par des Indiens, c'est-à-dire par les habitants du pays qui figure l'origine lointaine et mystérieuse du peuplement majoritaire de Maurice. Il s'agit de faire en sorte que les Indiens eux aussi désormais voient « double ", autrement dit de leur faire subir une opération de décentrement qui enregistrerait la reconnaissance du fait qu'il y a, non pas un, mais «deux Bénarès» (Ibid. : respectivement 77 et 79). Car de la même manière qu'il y a Paris (Texas), il y a Bénarès (Maurice) - artefact, village second d'une ville originelle qui a oublié ses enfants partis au loin et dont le fantôme vient hanter les vivants. Ce que voit surtout le narrateur dans le Bénarès indien, ce n'est en effet pas la vie qui grouille, mais "des mourants [...] vieux et malades pour la plupart, et pauvres", mourants qu'on traite « comme des chiens » (Ibid. : 68-69) et qui sont moins les mourants de l'Inde que les Indiens engagés en Inde et partis il y a longtemps à l'île Maurice, dont il ne reste rien que cette cheminée qui finit de signer leur oubli, et qui errent tels des fantômes en manque de monument. L'absence de lieu signe le lieu d'une absence - la dissolution de l'espace autour de la voiture signifiant en fait une dissolution du passé et l'envoûtement du narrateur par des présences spectrales. importante dans la littérature d'expression française de l'océan Indien, qu'on envisage 
l'île Maurice, les Comores, La Réunion ou Madagascar. La perte de la réalité que traduit la présence des fantômes est en fait une perte d'identité profondément liée à l'histoire de Maurice et d'une émigration indienne que caractérisent, comme l'explique bien Valérie Magdelaine-Andrianjafitrimo, « des histoires individuelles en lambeaux, agrégées en des histoires collectives fragmentées » et, du même coup, «la hantise du manque » (2006: 195). Mais là où, selon elle, les écrivains de Maurice et de La Réunion élaboreraient «la construction de récits des origines, de récits visant à restaurer la filiation, l'ancestralité, la fondation, tant familiale, individuelle, que collective» (Ibid. : 196), Barlen Pyamootoo ne mobilise pas des récits ou des figures ancrés dans les croyances traditionnelles et les fonds légendaires. C'est de manifestations plus discrètes et surtout moins précisément référencées qu'il s'agit - renforcement encore, d'une certaine manière, de leur propre nature fantomatique. Il n'est que de comparer Bénarès et Histoire d'Ashok et d'autres personnages de moindre importance du romancier Amal Sewtohul. Dans le récit de ce dernier, Vassou est guidé par l'âme de son grand-père qui lui apparaît périodiquement. Il traverse avec lui les éléments et les corps, vole, parcourt une myriade de lieux différents, connaît une série de métamorphoses et rencontre des âmes qui lui permettent de redécouvrir ses origines familiales et finalement de trouver « sa propre voie » (Sewtohul, 2002 : 169). Dans le récit de Barlen Pyamootoo, le récit du passé victimaire ne trouve pas sa résolution par l'intermédiaire d'un nouveau récit mémoriel garanti par la figure apaisante et réparatrice d'un ancêtre doté d'une dimension héroïque. La filiation reste défaillante, en tous les cas mal éclaircie, et les personnages ne sont projetés vers nul avenir assuré.

Il y a bien un «nous » qui se constitue, et l'on peut suivre encore Valérie MagdelaineAndrianjafitrimo, quand elle écrit que "ce "nous" ainsi constitué s'oppose aux autres, ceux qui ont voulu spolier l'île de sa créolisation comme les esclavagistes, les dominants de toutes les origines qui ne cessent de mentir sur l'histoire» (2006: 211). Le voyage en Inde est certes un voyage amont qui autorise que se remette en route, vers l'aval, la roue du temps, que s'ouvrent d'autres routes - vers Bénarès (Maurice) aussi bien que Bagdad (Irak). Il faut toutefois préciser une nouvelle fois que ce "nous» est réduit au seul habitacle de la voiture, à la seule communauté des lecteurs, et témoigne donc d'une réparation collective fort limitée à la fois dans le temps et dans l'espace. Soit que la souffrance du passé clandestin soit si grande qu'elle demeure indicible, soit - et nous privilégions cette hypothèse - qu'elle soit au contraire estompée et laisse le terrain libre à une élaboration esthétique détachée du souci de l'origine ou de l'injonction créolisante, les romans de Barlen Pyamootoo témoignent moins d'une quête de racines ou d'identités idéalisées que de l'invention sublimatoire d'un univers romanesque. Ils ne sont pas la simple transposition littéraire d'un imaginaire collectif d'ici ou de là-bas : ni restauration communautaire, ni pittoresque orientaliste. Ils imposent l'envoûtement d'une certaine manière d'être au monde, peut-être universellement partageable, sur le mode d'une présence à la fois ténue et intense. Fantômes, il y a - et fantômes d'un passé très précis ; mais fantômes que le texte fait aussi surgir de lui-même et qui rappellent à chacun - nul besoin d'être mauricien - les présences spectrales qui hantent sa propre histoire.

Les récits de Barlen Pyamootoo doivent beaucoup à la tradition minimaliste française - et cela limite au premier abord leur pouvoir de contestation du centre parisien. Ils ne sont pas comme À l'angle malang. Les Maux d'ici du Réunionnais Jean Louis Robert ou Za du Malgache Raharimanana fondés sur l'invention d'une langue apte à dire, chacune à leur manière et selon leur créativité propre, la complexité des mouvements de construction et 
de déconstruction identitaires à l'œuvre dans des îles au peuplement essentiellement et depuis toujours hétérogène ${ }^{10}$. On ne trouve ainsi dans Bénarès et dans Le Tour de Babylone aucun mot ou presque d'origine tamoule ou créole ni aucune néologie. Il est même possible que les présences fantomatiques de l'œuvre aient à voir avec le sentiment postmoderne de la répétitivité et donc de l'inutilité de toute création artistique. Tout a déjà été dit, et de toutes les façons, et l'écrivain mauricien contemporain - l'écrivain français aussi bien - a beau affirmer son individualité, celle-ci ne fait que retrouver et mimer des figures appartenant au passé et des histoires déjà jouées. Vertige, pour tout écrivain embarqué dans le monde d'aujourd'hui, des paysages culturels interchangeables et des siècles compilés derrière les vitres de la voiture - de l'histoire globalisée.

Le choix d'une langue s'est sans aucun doute posé pour Barlen Pyamootoo comme il se pose pour l'ensemble des écrivains francophones vivant en situation de diglossie sociale et dotés pour cette raison de ce que Lise Gauvin nomme une " surconscience linguistique ${ }^{11}$ " (2001: 153). Mais si l'écrivain a fait dans ses deux romans le choix du français et d'une certaine tradition littéraire, ce n'est sans doute pas uniquement pour atteindre le public éloigné de la France et recevoir une reconnaissance venue du centre ${ }^{12}$. Ce choix lui permet en effet de faire acte créatif d'une autre manière, tout aussi subversive que celle de la créolisation linguistique: par une langue minimaliste inattendue de ce côté du monde et déroutante par les représentations qu'elle élabore ${ }^{13}$. Les récits de Barlen Pyamootoo racontent des voyages qui ne conduisent pas vers le centre mais s'en affranchissent ostensiblement. On a beau être mauricien, on n'est pas nécessairement tourné vers la France, mais vers l'Inde ou l'Irak - relation de la périphérie à la périphérie ou, mieux encore, dissolution de cette polarisation unique pour une multiplication des pôles d'attraction permettant de préserver une distance et de se maintenir en lisière dans l'équilibre des forces. Loin de l'histoire et de ses mises en image ou en spectacle hiérarchisantes, de la violence symbolique des idéalisations mensongères, il s'agit, non pas de consentir à une identité périphérique originaire mais de faire du regard périphérique une destination; non pas de pleurer l'appartenance à la périphérie ou de la mettre en scène de manière pittoresque, mais d'adopter son point de vue et de se périphériser soi-même en quittant son village ou son pays et en s'ouvrant à la pluralité du monde et à sa propre pluralité intérieure. "Je me suis dit que c'est sûrement pour des histoires qu'on voyage, pour avoir quelque chose à raconter aux gens qu'on aime " (Pyamootoo, 1999: 70) : non pas le voyage lui-même, mais ce qu'à partir de lui on sent et on imagine; non pas la vérité passée ou présente - chromos du pouvoir et restauration douteuse -, mais un amalgame de sensations, de sentiments et de souvenirs; non pas l'un, mais son double : de la littérature.

Barlen Pyamootoo est né et vit à Maurice. Il est écrivain.

\section{BIBLIOGRAPHIE}




\section{Corpus principal}

Pyamootoo, Barlen, 1999, Bénarès, Paris, Éditions de l'olivier.

-, 2002, Le Tour de Babylone, Paris, Éditions de l'olivier.

-, 2008, Salogi's, Paris, Éditions de l'olivier.

\section{Corpus secondaire}

Appanah-Mouriquand, Nathacha, 2003, Les Rochers de Poudre d'Or, Paris, Gallimard, coll. « Continents noirs ».

Bouvier, Nicolas, 1992 [1963], L'Usage du monde, Paris, Payot, coll. « Petite Bibliothèque ».

Cabon, Marcel, 1981 [1965], Namasté, Rose Hill (Maurice), Éditions de l'océan Indien.

Devi, Ananda, 1989, Rue La Poudrière, Abidjan (Côte d'Ivoire), Nouvelles Éditions Africaines.

Humbert, Marie-Thérèse, 1979, À l'autre bout de soi, Paris, Stock.

Lacpatia, Firmin, 1978, Boadour. Du Gange... à la rivière des Roches (1861-1863), Saint-Denis (La Réunion), s. n.

Moutoussamy, Ernest, 1994, Chacha et Sosso, Paris, L'Harmattan, coll. « Lettres des Caraïbes ».

-, 1995 [1987], Aurore, Paris, L'Harmattan, coll. « Lettres des Caraïbes ».

Patel, Shenaz, 2005, Le Silence des Chagos, Paris, Éditions de l'olivier.

Raharimanana, 2008, Za, Paris, Éditions Philippe Rey.

Rahimi, Atiq, 2000 [1999], Terre et cendres, Paris, POL.

Rimbaud, Arthur, 1972 [1873], Une saison en enfer, dans Cuuvres complètes, édition établie et annotée par Antoine Adam, Paris, Gallimard, coll. « Bibliothèque de la Pléiade », p. 91-117.

Robert, Jean Louis, 2004, À l'angle malang. Les Maux d'ici, Saint-Denis (La Réunion), Éditions Grand Océan.

Sewtohul, Amal, 2001, Histoire d'Ashok et d'autres personnages de moindre importance, Paris, Gallimard, coll. « Continents noirs ».

Unnuth, Abhimanyu, 2001 [1977], Sueurs de sang, Paris, Stock, coll. « La Cosmopolite ».

\section{Autres références}

Benjelloun, Sandrine, 2007, « Le créole chagossien, marqueur identitaire d'une quête territoriale », dans Manuel Bengoéchéa, Delphine Chaume, Claire Riffard et Katerina Spiropoulou (dir.), Itinéraires et contacts de cultures, « Discours et écritures dans les sociétés en mutation », vol. 39, Paris, L'Harmattan / Université Paris 13, p. 105-121.

Bhabha, Homi K., 2007 [1994], Les lieux de la culture. Une théorie postcoloniale, traduit de l'anglais (États-Unis) par Françoise Bouillot, Paris, Payot.

Freud, Sigmund, 1985 [1919], « L'inquiétante étrangeté », dans L'Inquiétante étrangeté et autres essais, Paris, Gallimard, coll. « Connaissance de l'inconscient », p. 209-263. 
Gauvin, Lise, 2001, « Une situation d'interlangue : les romans d'Axel Gauvin », dans Kumari R. Issur et Vinesh Y. Hookoomsing, (dir.), 2001 : 153-166.

Hazareesingh, Kissoonsingh, 1973, Histoire des Indiens à l'île Maurice, Paris, Librairie d'Amérique et d'Orient.

Hookoomsing, Vinesh Y., 2001, «Crédité, coolitude : contextes et concepts », dans Kumari R. Issur et Vinesh Y. Hookoomsing (dir.), $2001: 251-264$.

Issur, Kumari R. et Hookoomsing, Vinesh Y. (dir.), 2001, L'océan Indien dans les littératures francophones. Pays réels, pays rêvés, pays révélés, actes du colloque de juillet 1997 organisé par l'université de Maurice, Paris, Karthala / Presses de l'université de Maurice.

Kundera, Milan, 1986, L'Art du roman, Gallimard.

Revue de Littérature comparée, 2006, «Les littératures indiaocéaniques », n³18, avril-juin.

Magdelaine-Andrianjafitrimo, Valérie, 2004a, « Une mise en scène de la diversité linguistique : comment la littérature francophone mauricienne se dissocie-t-elle des nouvelles normes antillaises?», Glottopol. Revue de sociolinguistique en ligne, « La littérature comme force glottopolitique : le cas des littératures francophones ", $\mathrm{n}^{\circ}$ 3, janvier, http://www.univ-rouen.fr/ dyalang/glottopol.

,$- 2004 b$, «"Ethnotexte" et intertextualité : la mise en scène des représentations culturelles dans les "romans ethnographiques" ", dans Jean-Claude Carpanin Marimoutou et Valérie MagdelaineAndrianjafitrimo, Univers créoles 4. Contes et romans, Paris, Anthropos, p. 93-145.

-, 2006, « Histoire et mémoire : variations autour de l'ancestralité et de la filiation dans les romans francophones réunionnais et mauriciens ", Revue de littérature comparée, 2006 : 195-212.

Marimoutou, Jean-Claude Carpanin, 2006, « Littératures indiaocéaniques », Revue de littérature comparée, 2006 : 131-140.

Ramharai, Vicram, 2006, « Le champ littéraire mauricien », Revue de littérature comparée, 2006 : 173-194.

Van den Avenne, Cécile, 2006, "L'élément indien de la "créolité" : une reconstruction identitaire ", dans Guillaume Bridet, Sarga Moussa, et Christian Petr (dir.), L'Usage de l'Inde dans les littératures française et européenne (XVIII-XX $\mathrm{X}^{e}$ siècles), Actes du colloque organisé à l'ENS Lettres \& Sciences Humaines (Lyon, 2-4 juin 2005), Paris-Pondichéry, Kailash, coll. « Cahiers de la SIELEC », p. 339-356.

\section{NOTES}

1. À la lecture de Bénarès, on pense à Duras quand il est fait mention de la ville de "Savannah " (80), et à Beckett quand Jimi s'exclame « Bénarès ! " et que le narrateur commente : « Ça a claqué comme une fin de partie » (90).

2. Sur la coolitude qu'initie Khal Torabully, voir Hookoomsing, 2001 : 256-263.

3. Pour tout ce qui concerne l'intégration de la composante indienne dans l'espace antillais francophone, voir Van den Avenne, 2006 : 339-356.

4. Le roman le plus connu d'Abhimanyu Unnuth, Sueurs de sang, constitue un véritable réquisitoire contre les crimes commis par les planteurs européens. L'intrigue, qui se déroule au milieu du XIX ${ }^{e}$ siècle, raconte la lutte désespérée menée par Kissan, jeune paysan hindou, contre le régime tout-puissant des planteurs. 
5. Dans son roman Les Rochers de Poudre d'Or, Nathacha Appanah-Mouriquand retrace l'arrivée d'un groupe d'Indiens à Maurice en 1892.

6. L'abolition de l'esclavage par la Chambre des Communes anglaise en 1833 nécessite l'arrivée d'une nouvelle main-d'œuvre bon marché venue des Indes.

7. Voir Benjelloun, 2007 : 116-117.

8. Contrairement à ce que peut renvoyer un certain cliché, la réalité de Maurice n'est pas apaisée de tout conflit communautaire. L'animosité se fonde sur l'opposition entre les cultures européenne et indienne, entre les langues européennes et asiatiques et le créole, entre différents modes de vie, etc. La dénonciation d'un "péril hindou »-et l'exacerbation des différences culturelles afférentes - est particulièrement présente à Maurice dans les années 1940-1950. Mais c'est dès le début $\mathrm{du} \mathrm{xx}^{\mathrm{e}}$ siècle que l'indianité suscite de très fortes peurs dans l'île, en raison notamment du poids du nombre sur lequel peut s'appuyer l'élite naissante d'origine indienne (voir Hazareesingh, 1973).

9. On peut prêter attention au surnom des deux mères maquerelles et les prendre au pied de la lettre. C'est conduits par un Jimi qui a l'âge d'être leur " grand-père » (Pyamootoo, 1999: 32), que les deux hommes se retrouvent devant la souteneuse « Ma Tante » qui leur suggère de se rendre chez « Maman » (Ibid., 25). Si la première est bien leur tante, cela signifie que sa maman est leur grand-mère et donc que les filles qu'elle leur procure pourraient être leur propre mère. S'expliquerait de la sorte, sous le regard de censeur d'une figure paternelle, le suspens de la consommation charnelle et son remplacement par des présences fantomatiques inquiétantes.

10. Il faut toutefois noter que, si la langue de Jean Louis Robert relève d'une forme de créolisation, celle de Jean-Luc Raharimanana dans $\mathrm{Za}$ est d'une tout autre nature. Elle cherche bien à produire des effets d'oralité, mais avec une inventivité plus personnelle. Il n'y a du reste pas eu de création d'un créole entre la langue malgache et la langue française.

11. Lorsque Barlen Pyamootoo adapte son roman Bénarès au cinéma en 2006, il décide de tourner le film en créole mauricien, ce qui constitue un signe on ne peut plus clair d'une réflexion sur la différence entre son lectorat et son public de spectateurs.

12. Ce désir ne serait en soi pas étonnant, tant les conditions de publication à Maurice sont difficiles. Outre que, depuis l'origine de la colonisation en 1715 et malgré la mainmise anglaise de 1810 à 1968 et la proclamation d'indépendance de 1968, le français reste la langue littéraire principale de l'île - essentiellement par le biais de l'instruction catholique et de la presse -, il faut attendre 2005 et la création des Éditions Bartholdi pour qu'une maison mauricienne se propose comme but principal d'encourager les écrivains mauriciens contemporains. Barlen Pyamootoo a lui-même contribué à favoriser ce type de publications dès 1994 par la création, avec Rama Poonoosamy, de la maison d'édition Immedia et, en particulier, de la collection " Maurice ", qui publie des anthologies thématiques de textes d'auteurs contemporains écrits principalement en français mais aussi en anglais et en créole (voir Ramharai, 2006 : 178-180).

13. Ce minimalisme n'est pas si rare et semble esquisser une nouvelle manière des littératures du Sud, peut-être parce qu'il constitue une neutralisation efficace des lieux communs exotiques. Voir par exemple, concernant non pas l'Irak, mais la guerre afghane, le très beau Terre et cendres (2000) d'Atiq Rahimi. La dimension subversive de ce type de démarche apparaît d'autant plus forte si l'on considère l'usage du français créolisé comme un signe d'identité normalisé et totalement intégré au marché littéraire français (voir Magdelaine-Andrianjafitrimo, 2004a: 142-165). 


\section{RÉSUMÉS}

Barlen Pyamootoo est un étrange écrivain mauricien qui semble venir de nulle part. Avant la publication de l'autobiographique Salogi's, il était en fait impossible de savoir de quel pays il venait en lisant ses livres et leurs titres, Bénarès et Le Tour de Babylone. Mais le but de son écriture est peut-être de déranger le lecteur et de le faire réfléchir sur le fait que les identités ne sont pas des réalités essentielles mais de véritables fictions, ce qui est précisément la révélation que nous pouvons attendre de la littérature.

Barlen Pyamootoo is a strange Mauritian writer, who seems to corne from nowhere. Before the publication of the autobiographical Salogi's, it was actually impossible to know what country he was from, when reading his books and their titles, Bénarès and Le Tour de Babylone. But his aim in writing is perhaps to disturb the reader and to make him think about the fact that identities are not essential realities but real fictions, which is precisely the revelation we can expect of literature.

\section{INDEX}

Mots-clés : île Maurice, Indiens, stéréotype, hybridité, fantastique

Keywords : Mauritius, Indians, hybridity, fantasy

\section{AUTEUR}

\section{GUILLAUME BRIDET}

Université Paris 13, CENEL 